\title{
Properties of Independently Axiomatizable Bimodal Logics
}

\author{
Marcus Kracht and Frank Wolter * \\ II. Department of Mathematics \\ Arnimallee 3 \\ 1000 Berlin 33 \\ GERMANY
}

\section{Introduction}

In mono-modal logic there is a fair number of high-powered results on completeness covering large classes of modal systems, witness for example Fine [74,85] and Sahlqvist [75]. Mono-modal logic is therefore a well-understood subject in contrast to poly-modal logic where even the most elementary questions concerning completeness, decidability etc. have been left unanswered. Given that so many applications of modal logic one modality is not sufficient, the lack of general results is acutely felt by the "users" of modal logics, contrary to logicians who might entertain the view that a deep understanding of modality alone provides enough insight to be able to generalize the results to logics with several modalities. Although this view has its justification, the main results we are going to prove are certainly not of this type, for they require a fundamentally new technique. The results obtained are called transfer theorems in Fine and Schurz [91] and are of the following type. Let $L \not \supset \perp$ be an independently axiomatizable bimodal logic and $L_{\square}$ as well as $L$ its mono-modal fragments. Then $L$ has a property $P$ iff $L_{\square}$ and $L_{\square}$ have $P$. Properties which will be discussed are completeness, finite model property, compactness, persistence, interpolation and Halldén-completeness. In our discussion we will show transfer theorems for the most simple case when there are just two modal operators but it will be clear that the proof works in the general case as well.

\section{Preliminaries}

Let $\mathcal{L}_{\square}$ be the language of bimodal logics with denumerably infinite propostional variables denoted by lower case Roman letters $p, q, \ldots$ and the primitive connectives $\wedge, \neg, \square$,

${ }^{*}$ We wish to thank Kit Fine, Valentin Goranko and Gerhard Schurz for discussing this paper with us and also the anonymous referee for carefully reading the manuscript. The completeness results have been obtained by Kit Fine and Gerhard Schurz independently and will be published in Fine and Schurz [91]. 
For a set $V$ of variables, $\mathcal{L}_{\square} \mathbf{\square}(V)$ is the sublanguage of formulae with variables from $V$. By $\mathcal{L}_{\square}$ we denote the fragment of $\boldsymbol{\square}$-free formulae, by $\mathcal{L}_{\square}$ the fragment of $\square$-free formulae. A set $L \subseteq \mathcal{L}_{\square}$ is called a normal (bimodal) logic if $L$ contains the axioms of classical logic, $B D^{\square}: \square(p \rightarrow q) . \rightarrow . \square p \rightarrow \square q$ and $B D^{\square}: \mathbf{\square}(p \rightarrow q) . \rightarrow . \square_{p} \rightarrow \mathbf{\square} q$ and which is closed under substitution, MP and $M N^{\square}: p / \square p, M N \square: p / \square p$. The minimal normal bimodal logic is denoted by $\mathbf{K}_{\square}$. If $L$ is a normal bimodal logic then $L_{\square}:=L \cap \mathcal{L}_{\square}$ and $L_{\square}:=L \cap \mathcal{L}_{\mathbf{\square}}$ are normal mono-modal logics. Conversely, given two mono-modal logics $M, N$ we can form the fusion $M \otimes N$ which is the least bimodal logic containing both $M$ and $N$ where the modal operator of $M$ is translated as $\square$ and the operator of $N$ by $\mathbf{\square}$. If $L=L_{\square} \otimes L_{\square}$ we call $L$ independently axiomatizable. Formally, there is a difference between $M \otimes N$ and $N \otimes M$, but exchanging $\square$ and $\boldsymbol{\square}$ induces an isomorphism from $M \otimes N$ to $N \otimes M$. We stress this point because there will quite often meet the situation that two statements are exactly the same if we exchange the modalities in one of the statements; we then say that one statement is the dual of the other. Given a bimodal logic $L$ we write $\Phi \vdash_{\square} \phi$ if $\phi$ can be deduced from $\Phi$ and the theorems of $L$ using Modus Ponens and the rules $\Phi \vdash_{\square} \phi \Rightarrow \square \Phi \vdash_{\square} \square \phi, \Phi \vdash_{\square} \phi \Rightarrow \square \Phi \vdash_{\square} \mathbf{\square} \phi$. We write $\Phi \vdash_{\square} \phi$ if $\phi$ can be deduced from $\Phi$ and the axioms of $L_{\square}$ using only Modus Ponens and the rule $\Phi \vdash_{\square} \phi \Rightarrow \square \Phi \vdash_{\square} \square \phi$. And likewise for the dual case.

Given a formula $\phi \in \mathcal{L}_{\square}$ we denote the set of subformulae of $\phi$ by $s f(\phi)$ and the set of variables by $\operatorname{var}(\phi)$. The modal degree $d g(\phi)$ of $\phi$ is defined by

$$
\begin{aligned}
d g(p) & =0 \\
d g(\neg \phi) & =d g(\phi) \\
d g(\phi \wedge \psi) & =\max \{d g(\phi), d g(\psi)\} \\
d g(\square \phi) & =d g(\phi)+1 \\
d g(\mathbf{\square} \phi) & =d g(\phi)+1
\end{aligned}
$$

The $\square$-degree $d g^{\square}(\phi)$ of $\phi$ is defined similarly with the exception that $d g^{\square}(\mathbf{\square} \phi)=$ $d g^{\square}(\phi)$, i.e. occurrences of $\boldsymbol{\square}$ are not counted. With an analoguous definition for $d g \mathbf{\square}_{(\phi)}$ we then have $d g(\phi) \leq d g^{\square}(\phi)+d g \mathbf{M}_{(\phi)}$. (Equality need not hold, e.g. $\square \square \square p \vee \square \square \square p$.) Suitable structures for interpreting $\mathcal{L}_{\square}$ are bimodal algebras, which are triples $\langle\mathbf{B}, \square, \mathbf{\square}\rangle$ such that $\langle\mathbf{B}, \square\rangle$ and $\langle\mathbf{B}, \mathbf{\square}\rangle$ are modal algebras, that is, boolean algebras $\langle B, \backslash, \cap\rangle$ with an operator $\square$ satisfying $\square 1=1$ and $\square(a \cap b)=\square a \cap \square b$. By standard representation theorems (Jónsson and Tarski [51]) bimodal algebras can be represented by generalized frames $\langle g, \triangleleft, \varangle, \mathbb{G}\rangle$ where $g$ is a set (e.g. the set of ultrafilters of $\mathbf{B}), \triangleleft, \triangleleft$ binary relations on $g$ and $\mathbb{G} \subseteq 2^{g}$ a system of sets closed under complementation, intersection and

$$
\begin{aligned}
& \square A:=\{s \mid \forall t(s \triangleleft t \rightarrow t \in A)\} \\
& \square A:=\{s \mid \forall t(s \triangleleft t \rightarrow t \in A)\}
\end{aligned}
$$

If $\mathbb{G}=2^{g}$ we write $\langle g, \triangleleft, \triangleleft\rangle$ instead of $\langle g, \triangleleft, \varangle, \mathbb{G}\rangle$ and call $\langle g, \triangleleft, \triangleleft\rangle$ a (bimodal) frame. A valuation on $\langle g, \triangleleft, \triangleleft, \mathbb{G}\rangle$ is a map $\beta: V \longrightarrow \mathbb{G}$ for a set $V$ of variables. The pair $\langle\langle g, \triangleleft, \triangleleft, \mathbb{G}\rangle, \beta\rangle$ is called a model. $\beta$ extends to a homomorphism $\bar{\beta}: \mathcal{L}_{\square}(V) \longrightarrow$ $\langle\mathbb{G}, \backslash, \cap, \square, \mathbf{\square}\rangle$. We write $\langle g, \triangleleft, \triangleleft, \mathbb{G}\rangle, \beta, s \models \phi$ for $s \in \bar{\beta}(\phi)$ and say that $\phi$ is true at $s$ in 
that model and we write $\langle g, \triangleleft, \triangleleft, \mathbb{G}\rangle, \beta=\phi$ for $g=\bar{\beta}(\phi)$. If for every valuation defined on $\operatorname{var}(\phi)\langle g, \triangleleft, \varangle, \mathbb{G}\rangle, \beta=\phi$ we say that the frame validates $\phi$ and write $\langle g, \triangleleft, \triangleleft, \mathbb{G}\rangle \models \phi$. In a frame $\langle g, \triangleleft, \boldsymbol{\triangleleft}\rangle$ we denote by $\operatorname{Tr}^{\square}(x, g)$ the set as well as the subframe generated by $x$ in $g$ following only the $\triangleleft$-relation.

\section{Some Useful Constructions}

Let $\mathcal{E} L$ denote the lattice of extensions of a modal logic. We have defined an operation $-\otimes-:(\mathcal{E} K)^{2} \rightarrow \mathcal{E} K_{\square} \mathbf{\square}$. $\otimes$ is a $\bigsqcup$-homomorphism in both arguments. There are certain easy properties of this map which are noteworthy. Fixing the second argument we can study the map $-\otimes M: \mathcal{E} K \rightarrow \mathcal{E} K_{\square}$. This is a $\sqcup$-homomorphism. The map $-_{\square}: \mathcal{E} K_{\square} \rightarrow \mathcal{E} K: L \mapsto L_{\square}$ will be shown to almost the inverse of $-\otimes M$. First, if $L$ is a normal modal logic then $(L \otimes M)_{\square} \supseteq L$. Similarly, for a normal bimodal logic $L, L_{\square} \otimes L \square \subseteq L . \quad$ For $\langle g, \triangleleft, \triangleleft, \mathbb{G}\rangle \models L$ implies $\langle g, \triangleleft, \mathbb{G}\rangle \models L_{\square}$ and $\langle g, \boldsymbol{\triangleleft}, \mathbb{G}\rangle \models L \square$. This implies in turn that $\langle g, \triangleleft, \varangle, \mathbb{G}\rangle \models L_{\square} \otimes L_{\mathbf{\square}}$. Consequently, if $L$ is independently axiomatizable then $\langle g, \triangleleft, \varangle, \mathbb{G}\rangle$ is a general $L$-frame iff $\langle g, \triangleleft, \mathbb{G}\rangle$ is a general $L_{\square}$-frame and $\langle g, \boldsymbol{\varangle}, \mathbb{G}\rangle$ is a general $L_{\mathbf{\square}}$-frame.

Theorem 1 (Thomason) $(L \otimes M)_{\square}=L$ iff $\perp \notin M$ or $\perp \in L$.

Proof. $(\Rightarrow)$ Suppose $\perp \in M$ and $\perp \notin L$. Then $\perp \in L \otimes M$ and hence $\perp \in(L \otimes M)_{\square}$, so hat $L \neq(L \otimes M)_{\square}$.

$(\Leftarrow)$ Suppose $\perp \in L$. Then $\perp \in L \otimes M$ and so $\perp \in(L \otimes M)_{\square}$ from which $L=(L \otimes M)_{\square}$. Now suppose $\perp \notin L$. Then $\perp \notin M$ and by a result of Makinson [71] either $\bullet \models M$ or $\mathbf{\mathrm { X }} \models M$. Let $\mathcal{G}=\langle g, \triangleleft, \mathbb{G}\rangle$ be an $L$-frame. Then define $\mathcal{G}^{\mathrm{\times}}=\langle g, \triangleleft, \triangleleft, \mathbb{G}\rangle$ by $\triangleleft:=\emptyset$ and $\mathcal{G}^{\bullet}=\langle g, \triangleleft, \varangle, \mathbb{G}\rangle$ by letting $\boldsymbol{\triangleleft}:=\{\langle x, x\rangle \mid x \in g\}$. It is readily checked that $\mathbf{\square} a=1$ in $\mathcal{G}^{\mathbf{x}}$ and $a=a$ in $\mathcal{G}^{\bullet}$ so that both are in fact general frames. If $\mathrm{X}=M$ then $\mathcal{G}^{\times}$is a $L \otimes M$ frame and if $\bullet \models M$ then $\mathcal{G}^{\bullet}$ is a $L \otimes M$-frame. For $\phi \in \mathcal{L}_{\square} \mathcal{G}=\phi \Leftrightarrow \mathcal{G}^{\times} \models \phi \Leftrightarrow \mathcal{G}^{\bullet} \models \phi$. Thus $(L \otimes M)_{\square} \subseteq L$ and therefore $(L \otimes M)_{\square}=L . \dashv$

This theorem is proved algebraically in Thomason [80]; a syntactic proof is given in Fine and Schurz [91]. The theorem states that if $L \in L$ or $\top \notin M$ then $L \otimes M$ is a conservative extension of $L$. Thus given two logics $L, M$ we have both $L=(L \otimes M)_{\square}$ and $M=(L \otimes M) \square$ iff $\perp \in L \Leftrightarrow \perp \in M$. In all the theorems that will follow we will therefore simply exclude the case that $\perp \in L$ or $\perp \in M$ which are trivial anyway. The way in which we used Makinson's theorem to build a minimal extension of a mono-modal frame to a bimodal frame is worth remembering. It will occur quite often later on. Although Makinson's theorem has no analogue for bimodal logics as there are infinitely many maximal consistent bimodal logics, at least for independemtly axiomatizable logics the following holds.

Corollary 2 Suppose that $L$ is a consistent independently axiomatizable bimodal logic. Then there is an L-frame based on one point. $\dashv$ 
Another immediate consequence concerns finite axiomatizablity, or f.a., for short. A logic $L$ is finitely axiomatizable if there is a finite set $X$ such that $L=\mathbf{K}(X)$.

Theorem 3 Suppose that $\perp \notin L, M$. Then $L \otimes M$ is finitely axiomatizable iff both $L$ and $M$ are.

Proof. Only the direction from left to right is not straightforward. Assume therefore that $L \otimes M$ is f.a., say $L \otimes M=\mathbf{K}_{\square} \mathbf{\square}(Z)$. If $L=\mathbf{K}_{\square}(X), M=\mathbf{K}_{\mathbf{\square}}(Y)$ then $Z \subseteq$ $\mathbf{K}_{\square} \mathbf{\square}(X \cup Y)$ and by Compactness Theorem we have finite sets $X_{0} \subseteq X, Y_{0} \subseteq Y$ such that $Z \subseteq \mathbf{K}_{\square} \mathbf{\square}\left(X_{0} \cup Y_{0}\right)$. But then $L \otimes M=\mathbf{K}_{\square} \mathbf{\square}\left(X_{0} \cup Y_{0}\right)=\mathbf{K}_{\square}\left(X_{0}\right) \otimes \mathbf{K}_{\square}\left(Y_{0}\right)$ and hence $L=\mathbf{K}_{\square}\left(X_{0}\right)$ and $M=\mathbf{K}_{\mathbf{\square}}\left(Y_{0}\right) . \dashv$

\section{Persistence is Invariant under Fusion}

Given a class $\mathcal{X}$ of bimodal general frames and a bimodal logic $L$ we say that $L$ is $\mathcal{X}$ persistent if for all $\langle g, \triangleleft, \triangleleft, \mathbb{G}\rangle \in \mathcal{X}\langle g, \triangleleft, \triangleleft, \mathbb{G}\rangle \models L$ implies $\langle g, \triangleleft, \triangleleft\rangle \models L$. A welcome property of persistence is that it is preserved by infinite joins. For suppose that $\langle g, \triangleleft, \triangleleft$ $, \mathbb{G}\rangle \models \bigsqcup\left\langle L_{i} \mid i \in I\right\rangle$. Then $\langle g, \triangleleft, \mathbf{4}, \mathbb{G}\rangle=L_{i}$ for every $i \in I$ from which $\langle g, \triangleleft, \triangleleft\rangle \mid=L_{i}$ for every $i \in I$, since the $L_{i}$ are $\mathcal{X}$-persistent; therefore $\langle g, \triangleleft, \triangleleft\rangle \models \sqcup\left\langle L_{i} \mid i \in I\right\rangle$. Now if $\mathcal{X}$ is a class of general bimodal frames, put $\mathcal{X}_{\square}:=\{\langle g, \triangleleft, \mathbb{G}\rangle \mid\langle g, \triangleleft, \triangleleft, \mathbb{G}\rangle \in \mathcal{X}\}$ and $\mathcal{X}_{\square}:=\{\langle g, \boldsymbol{\varangle}, \mathbb{G}\rangle \mid\langle g, \triangleleft, \boldsymbol{\varangle}, \mathbb{G}\rangle \in \mathcal{X}\}$. Then if $L_{\square}$ is $\mathcal{X}_{\square}$-persistent and $L_{\square}$ is $\mathcal{X}_{\square}$-persistent, $L_{\square} \otimes L_{\square}$ is $\mathcal{X}$-persistent. For suppose that $\langle g, \triangleleft, \varangle, \mathbb{G}\rangle \models L_{\square} \otimes L_{\square}$ and $\langle g, \triangleleft, \triangleleft, \mathbb{G}\rangle \in \mathcal{X}$. Then both $\langle g, \triangleleft, \mathbb{G}\rangle \models L_{\square}$ with $\langle g, \triangleleft, \mathbb{G}\rangle \in \mathcal{X}_{\square}$ and $\langle g, \triangleleft, \mathbb{G}\rangle \models L_{\square}$ with $\langle g, \triangleleft, \mathbb{G}\rangle \in \mathcal{X}_{\mathbf{\square}}$. Then $\langle g, \triangleleft\rangle \models L_{\square}$ and $\langle g, \triangleleft\rangle \models L_{\square}$ from which $\langle g, \triangleleft, \triangleleft\rangle \mid=L_{\square} \otimes L_{\square}$.

In modal logic, two classes of general frames play an important role with respect to persistence, namely the class $\mathcal{R}$ of refined frames and the class $\mathcal{D}$ of descriptive frames. A bimodal general frame $\langle g, \triangleleft, \varangle, \mathbb{G}\rangle$ is called refined if it satisfies

$$
\begin{aligned}
& \text { (df) } \quad \forall s, t \in g(s=t \leftrightarrow \forall a \in \mathbb{G}(s \in a \leftrightarrow t \in a)) \\
& (t \square) \quad \forall s, t \in g(s \triangleleft t \leftrightarrow \forall a \in \mathbb{G}(s \in \square a \rightarrow t \in a)) \\
& (t \square) \quad \forall s, t \in g(s \triangleleft t \leftrightarrow \forall a \in \mathbb{G}(s \in \mathbf{\square} a \rightarrow t \in a))
\end{aligned}
$$

If $\langle g, \triangleleft, \triangleleft, \mathbb{G}\rangle$ is refined as a bimodal frame, then the mono-modal reducts are refined as mono-modal frames. A general frame $\mathcal{G}=\langle g, \triangleleft, \varangle, \mathbb{G}\rangle$ is called descriptive if the map which sends $x \in g$ to the uniquely determined ultrafilter $x^{b}$ satisfying $\bigcap x^{b} \ni x$ is an isomorphism between $\mathcal{G}$ and its bidual $\mathcal{G}^{b}$ which we obtain as follows. Take $g^{b}$ to be the set of all ultrafilters of $\langle\mathbb{G}, \backslash, \cap\rangle$ and for $a \in \mathbb{G}$ put $a^{b}=\left\{U \in g^{b} \mid a \in U\right\}$. Now define

$$
\begin{aligned}
& U \triangleleft^{b} T \Leftrightarrow \forall \square a \in U(a \in T) \\
& U \varangle^{b} T \Leftrightarrow \forall \square a \in U(a \in T) \\
& \mathbb{G}^{b}=\left\{a^{b} \mid a \in \mathbb{G}\right\}
\end{aligned}
$$

Then $\mathcal{G}^{b}=\left\langle g^{b}, \triangleleft^{b}, \triangleleft^{b}, \mathbb{G}^{b}\right\rangle$ is the bidual of $\mathcal{G}$. Again, if $\mathcal{G}$ is descriptive as a bimodal frame 
then its mono-modal frames are descriptive as mono-modal frames. Therefore we have the following results.

Theorem 4 Suppose that $\perp \notin L, M$. Then $L \otimes M$ is $\mathcal{R}$-persistent iff both $L$ and $M$ are $\mathcal{R}$-persistent.

Proof. Suppose that $\perp \notin L$ is not $\mathcal{R}$-persistent. We have to show that $L \otimes M$ is also not $\mathcal{R}$-persistent. We know that there is an $L$-frame $\mathcal{G}=\langle g, \triangleleft, \mathbb{G}\rangle$ such that $g \not \models L$. On the condition that both $\mathcal{G}^{\times}$and $\mathcal{G}^{\bullet}$ are both refined, the theorem is proved. For either $\mathcal{G}^{\times} \models L \otimes M$ or $\mathcal{G}^{\bullet}=L \otimes M$, but $\langle g, \triangleleft, \triangleleft\rangle \not \models L \otimes M$ since $\langle g, \triangleleft\rangle \not \models L$.

Both $\mathcal{G}^{\times}$and $\mathcal{G}^{\bullet}$ satisfy $(d f)$ and $(t \square)$. That $\mathcal{G}^{\bullet}$ satisfies $(t \boldsymbol{\square})$ is seen as follows. If $s=t$ then for all $a \in \mathbb{G}, s \in \mathbf{\square} a$ implies $s \in a$ since $\mathbf{\square} a=a$. But if $s \neq t$ there is a $a \in \mathbb{G}$ such that $s \in a, t \notin a$. Then $s \in \boldsymbol{\square} a, t \notin a$, as required. Similarly, $\mathcal{G}^{\times}$satisfies $(t \boldsymbol{\square})$ since for arbitrary $s, t$ there is $a \in \mathbb{G}$ with $t \notin a$. Then $s \in \mathbf{\square} a, t \notin a$, since $\mathbf{\square} a=1$. $\dashv$

Theorem 5 Suppose that $\perp \notin L, M$. Then $L \otimes M$ is $\mathcal{D}$-persistent iff both $L$ and $M$ are $\mathcal{D}$-persistent.

Proof. As in the previous theorem. One only has to check that if $\mathcal{G}$ is descriptive, so are $\mathcal{G}^{\times}$and $\mathcal{G}^{\bullet}$. This is routine. $\dashv$

Descriptive frames are exactly the frames which are representations of modal algebras. We call a frame $\mathcal{G}$ a canonical frame for $L$ if it is the representation of a freely $\alpha$-generated $L$-algebra, where $\alpha$ is a cardinal number. Then $L$ is canonical if it is persistent with respect to its canonical frames.

Corollary 6 Suppose that $\perp \notin L, M$. Then $L \otimes M$ is canonical iff both $L$ and $M$ are canonical.

Proof. By a theorem of Sambin and Vaccaro [88] a modal logic is canonical iff it is $\mathcal{D}$-persistent. $\dashv$

\section{The Fundamental Theorem}

$L$ is complete if $\nvdash \phi$ iff there is a $L$-frame $\langle g, \triangleleft, \mathbf{\triangleleft}\rangle \not \models \phi$ and $L$ has f.m.p. iff $\nvdash \phi$ is equivalent to $\langle g, \triangleleft, \mathbf{\triangleleft}\rangle \not \models \phi$ for some finite $L$-frame $\langle g, \triangleleft, \mathbf{4}\rangle$. Given that $L$ is complete (has f.m.p.) it is immediate that both $L_{\square}$ and $L_{\square}$ are complete (have f.m.p.). Therefore, if $L, M$ are mono-modal logics and $\perp \notin L, M$ then completeness of $L \otimes M$ implies completeness of $L$ and $M$.

Theorem 7 Suppose $\perp \notin L, M$. Then $L \otimes M$ is complete iff both $L$ and $M$ are complete. 
We will first give the reader an idea of how the proof works in principle. Suppose we want to show that $\mathbf{K B} \otimes \mathbf{K B}$ has the finite model property, where $\mathbf{K B}=\mathbf{K}(p \rightarrow \square \diamond p)$. Let us try the formula $P=\diamond \square(p \wedge \checkmark \mathbf{\square} \square p)$. This formula is consistent and we should be able to produce a finite model for it. Since we only know how to build KB-models, we construct a model for $P$ stepwise. In the first step we treat all maximal subformulas of type $Q$ as variables, which we denote by $q \triangleleft Q$. This yields the formula $P^{\square}=\diamond \square(p \wedge q \triangleleft \square \diamond \square p)$. For this formula we can build a KB-model.

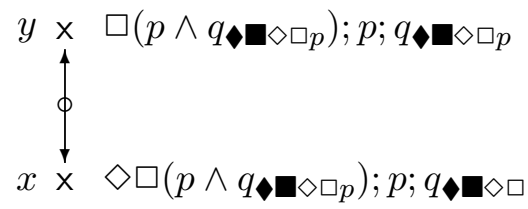

Here, $x$ denotes an irreflexive point and $\circ$ indicates the $\triangleleft$-arrows, while the $\triangleleft$-arrows will be denoted by •. Our task is obviously not finished as now each point contains these complex variables which can be viewed as placeholders for models which are yet to be built. Since the logic is independent in both modalities we can treat each point separately. For every point, a model for the formula $p \wedge \diamond \mathbf{\square} \square p$ has to be built and to be tagged onto the existing model at that point. The construction will now be dual to the previous one: we now replace maximal subformulas of type $\diamond Q$ by variables $q \diamond Q$.

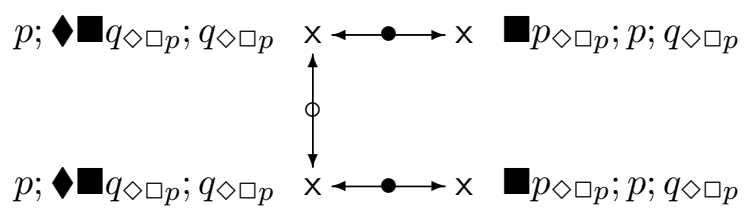

Finally, at each of the four points we have to build a model for $\diamond \square p$. At $x$ and $y$, which are on the left, this formula is already satisfied. At the other two points we glue a $\triangleleft$-reflexive one-point frame (denoted by o).

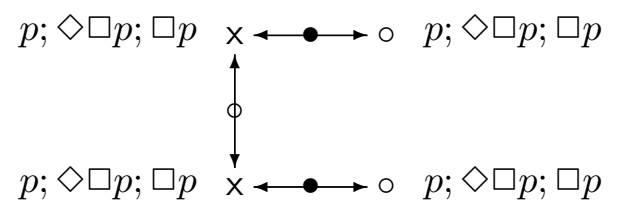

There are several ways in which this construction might have gone wrong. First, we might have chosen the following model in the first step.

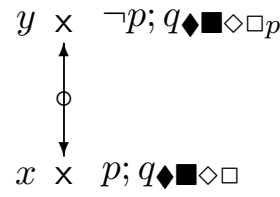


But since $\diamond \mathbf{\square} \diamond \square \vdash_{\mathbf{K B} \otimes \mathbf{K B}} p$ we would not be able to complete the construction, since in the third step the model will "backfire" on $y$ forcing $y \models p$; we will avoid this by working with partial valuations which only assign values if necessary. Second, even though we work with partial valuations the same conflict might arise e.g. if we chose the wrong frame to start with. We preempt such difficulties by adding to $P^{\square}$ a "consistency"-formula which makes sure that within a certain distance from $x$ all valuations are $\mathbf{K B} \otimes \mathbf{K B}$-consistent; by going partial this will be enough to be sure that our construction never backfires. The consistency formulae have to be chosen very carefully in order to avoid the above difficulties and others which occur if the construction of the model needs several iterations.

As we have noted, the direction from left to right is straightforward and so we will concentrate on the other direction. For each formula $\square \psi, \boldsymbol{\square} \psi \in \mathcal{L}_{\square}$ we reserve a variable $q_{\square \psi}$ and $q \square \psi$ respectively, which we call the surrogate of $\square \psi(\square \psi) . \quad q_{\square \psi}$ is called a $\square$-surrogate and $q \square \psi$ a distinct from our original set of variables. Any variable which is not a surrogate is called a p-variable and every formula composed exclusively from $p$-variables a p-formula. A $p$-variable is denoted by $p, p_{1}, \ldots, p_{i}, \ldots$ and an arbitrary variable by $q$. Finally, if $\phi$ is a formula, then $\operatorname{var}^{p}(\phi)$ denotes the set of $p$-variables of $\phi$, and likewise the $\operatorname{var}^{\square}(\phi), \operatorname{var}^{\mathbf{\square}}(\phi)$ denote the set of $\square$-surrogates of $\phi$ and the set of $\boldsymbol{\square}$-surrogates. The set of $p$-variables in $\mathcal{L}_{\square}$ is assumed to be countably infinite.

Definition 8 For a p-formula $\phi$ we define the $\square$-ersatz $\psi^{\square} \in \mathcal{L}_{\square}$ of $\psi$ as follows:

$$
\begin{aligned}
q^{\square} & =q \\
\left(\psi_{1} \wedge \psi_{2}\right)^{\square} & =\psi_{1}^{\square} \wedge \psi_{2}^{\square} \\
(\neg \psi)^{\square} & =\neg \psi^{\square} \\
(\square \psi)^{\square} & =\square \psi^{\square} \\
(\boldsymbol{\square} \psi)^{\square} & =q \mathbf{\square} \psi
\end{aligned}
$$

For a set $\Gamma$ of $p$-formulae call $\Gamma^{\square}=\left\{\psi^{\square} \mid \psi \in \Gamma\right\}$ the $\square$-ersatz of $\Gamma$. Dually for

Now let $\psi$ be composed either without $\square$-surrogates or without $\square$-surrogates. Then we define the reconstruction $\uparrow \psi$ of $\psi$ as follows.

$$
\begin{aligned}
& \uparrow \psi=\psi\left(\mathbf{\square} \psi_{1}^{\mathbf{\square}} / q_{\mathbf{\square}}, \ldots, \psi_{1}, \ldots, q_{\ell} / q_{\ell}, p_{1}, \ldots, p_{m}\right) \\
& \uparrow \psi=\psi\left(\square \psi_{1}^{\square} / q_{\square \psi_{1}}, \ldots, \square \psi_{\ell}^{\square} / q_{\square \psi_{\ell}}, p_{1}, \ldots, p_{m}\right)
\end{aligned}
$$

Note that if $\uparrow$ is defined on $\psi$ it is also defined on $\uparrow \psi$; for if $\psi$ was free of $\square$-surrogates, $\uparrow \psi$ is free of $\boldsymbol{\square}$-surrogates and vice versa. Now if $\phi$ is a $p$-formula then $\phi^{\square}$ is free of $\square$ surrogates and therefore the reconstruction operator is defined on $\phi$. Also, if $\uparrow$ is defined on $\psi$ then for some $n \in \omega \uparrow^{n+1} \psi=\uparrow^{n} \psi$ (where $\uparrow^{n}$ denotes the $n$th iteration of $\uparrow$ ) which is the case exactly if $\uparrow^{n} \psi$ is a $p$-formula. We then call $\uparrow^{n} \psi$ the total reconstruction of $\psi$ and denote it by $\psi^{\uparrow} \cdot \psi^{\uparrow}$ results from $\psi$ by replacing each occurrence of a surrogate $q_{\chi}$ in 
$\psi$ by $\chi$. Now let $\phi$ be a $p$-formula. Then we define $\phi_{n}=\uparrow^{n}\left(\phi^{\square}\right)$. It is clear that $\phi^{\square \uparrow}=\phi$. The $\square$-alternation-depth of $\phi-a d p^{\square}(\phi)$ - is defined by $a d p^{\square}(\phi)=\min \left\{n \mid \phi_{n}=\phi\right\}$. For $m>a d p^{\square}(\phi), \phi_{m}=\phi_{m-1}$. The -alternation depth, $a d p(\phi)$ is defined dually and $a d p(\phi)=\left(a d p^{\square}(\phi)+a d p(\phi)\right) / 2$. It is easy to show that $\left|a d p^{\square}(\phi)-a d p(\phi)\right| \leq 1$. For example, if $\phi \in \mathcal{L}_{\square}$ then $a d p^{\square}(\phi)=0$ and $a d p(\phi)=1$ and so $a d p(\phi)=1 / 2$. Conversely, $\operatorname{adp}(\phi)=1 / 2$ implies $\phi \in \mathcal{L}_{\square} \cup \mathcal{L}$

Definition 9 Let $L$ be a (bimodal) logic and $\Delta \subseteq \mathcal{L}_{\square}$ be a finite set. Then the consistency formula $\Sigma(\Delta)$ of $\Delta$ (with respect to $L$ ) is defined by $\Sigma(\Delta)=\bigvee\left\langle\psi_{c} \mid c \in C\right\rangle$, where $\psi_{c}=\bigwedge\langle\chi \mid \chi \in c\rangle \wedge \bigwedge\langle\neg \chi \mid \chi \notin c\rangle$ and $C=\left\{c \subseteq \Delta \mid \psi_{c}\right.$ is L-consistent $\}$. If $\Delta$ is an infinite set then we define the consistency set $\Sigma(\Delta)$ of $\Delta$ to be $\Sigma(\Delta):=\left\{\Sigma\left(\Delta^{\prime}\right) \mid \Delta^{\prime} \subseteq\right.$ fin $\left.\Delta\right\}$.

Note that the consistency formulas are $L$-theorems. We abbreviate the consistency formula for the set $s f\left\{\psi \mid q_{\psi} \in \operatorname{var}\left(\phi^{\square}\right)\right\} \cup \operatorname{var}^{p}(\phi)$ by $\Sigma_{\square}(\phi)$. In the proof of Theorem 7 we construct not ordinary models but partial models. If $g$ is a frame and $V$ a set of variables then $\beta: V \rightarrow\{0,1, *\}^{g}$ is called a partial valuation. Here, 0,1 are called the standard truth values and $*$ is the undefined or - to avoid confusion - the nonstandard truth value. We define the value of a formula according to the three-valued logic of 'inherent undefinedness'. It has the following truth tables

\begin{tabular}{|c|c|c|c|c|c|}
\hline & $\neg$ & $\wedge$ & 0 & 1 & $*$ \\
\hline 0 & 1 & 0 & 0 & 0 & $*$ \\
\hline 1 & 0 & 1 & 0 & 1 & $*$ \\
\hline$*$ & $*$ & $*$ & $*$ & $*$ & $*$ \\
\hline
\end{tabular}

We define $\bar{\beta}(\neg \phi, x)=\neg \bar{\beta}(\phi, x), \bar{\beta}(\phi \wedge \psi, x)=\bar{\beta}(\phi, x) \wedge \bar{\beta}(\psi, x)$ and $\bar{\beta}(\square \phi, x)=\bigwedge\langle\bar{\beta}(\phi, y)| x \triangleleft$ $y\rangle$. Note that by definition $\diamond \phi$ and $\square \phi$ receive a standard truth value iff every successor receives a standard truth value. We define the following order on the truth values

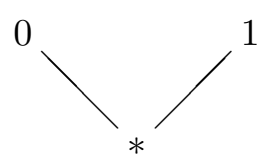

In the sequel we will assume that all valuations are defined on the entire set of variables. In contrast to what is normally considered a partial valuation, namely a partial function from the set of variables, the source of partiality or undefinedness is twofold. It may be local, when a variable or formula fails to be standard at a single world, or global, when a variable or formula is nonstandard throughout a frame. Our proof relies crucially on the ability to allow for local partiality. The domain of a valuation $\beta: V \rightarrow\{0,1, *\}^{g}$ is the set of variables on which $\beta$ is globally partial i.e. $\operatorname{dom}(\beta):=\{q \mid(\exists x \in g) \beta(q, x) \neq *\}$. If $\beta, \gamma: V \rightarrow\{0,1, *\}^{g}$ we define $\beta \leq \gamma$ if $\beta(p, x) \leq \gamma(p, x)$ for all $p \in V$ and all $x \in g$. It is easy to see that if $\beta \leq \gamma$ then for all $x \in g$ and all $\phi$ with $\operatorname{var}(\phi) \subseteq V: \bar{\beta}(\phi, x) \leq \bar{\gamma}(\phi, x)$. 
Hence if $\beta$ and $\gamma$ are comparable then they assign equal standard truth values to formulas to which they both assign a standard truth value. In the proof we will only have the situation where a partial valuation $\beta$ is nonstandard either on all $\square$-surrogates or on all

- -surrogates. In the latter case we define for a point $x \in g$ and a set $\Delta$ of formulae

$$
\begin{aligned}
& X_{\square}^{\beta, \Delta}(x)=\quad\left\{\psi \mid \psi \in \Delta, \bar{\beta}\left(\psi^{\square}, x\right)=1\right\} \\
& \cup \quad\left\{\neg \psi \mid \psi \in \Delta, \bar{\beta}\left(\psi^{\square}, x\right)=0\right\}
\end{aligned}
$$

and call $X_{\square}^{\beta, \Delta}(x)$ the characteristic set of $x$ in $\langle g, \beta\rangle$. If $X_{\square}^{\beta, \Delta}(x)$ is finite (for example, if $\Delta$ is finite), then $\chi_{\square}^{\beta, \Delta}(x)=\bigwedge X_{\square}^{\beta, \Delta}(x)$ is the characteristic formula of $x$. And dually $X_{\mathbf{m}}^{\beta, \Delta}(x)$ and $\chi_{\mathbf{m}}^{\beta, \Delta}(t)$ are defined. We call a set $\Delta$ sf-founded if for all $\chi \in \Delta$ and $\tau \in \operatorname{sf}(\chi)$ then either $\tau \in \Delta$ or $\neg \tau \in \Delta$.

Before we begin the proof of the theorems, let us agree on some abbreviations. If $\langle g, \triangleleft\rangle$ is a frame and $x, y \in g$, write $\operatorname{dist}^{\square}(x, y)=k$ if $k$ is the smallest number such that there is a sequence $\left\langle x_{i} \mid i \in k+1\right\rangle$ with $x_{0}=x, x_{k}=y$ and $x_{i} \triangleleft x_{i+1}$ for all $i \in k$. Also write $\square^{(k)} \phi=\bigwedge\left\langle\square^{\ell} \phi \mid \ell \leq k\right\rangle$. If $x \in g$ and $\beta$ is a partial valuation then if for all proper subformulas $\psi$ of $\phi \psi$ is defined on all points $y$ with $d g^{\square}(\psi)+d i s t^{\square}(x, y) \leq d g^{\square}(\phi)$ then $\phi$ is defined at $x$. This is proved by induction on $\phi$. Finally, if $g$ and $h$ are Kripke-frames, their disjoint union is denoted by $g \oplus h$.

Proof of Theorem 7: Assume $\nvdash_{\square} \neg \phi$ and $a d p^{\square}(\phi)=n$. Denote by $\mathbb{S}_{i}$ the set $s f\left\{\psi \mid q_{\psi} \in\right.$ $\left.\operatorname{var}\left(\phi_{i}\right)\right\} \cup \operatorname{var}^{p}(\phi)$. For $i=0$ this is exactly the set of formulas on which the consistency formulas for $\phi$ is defined. We will use an inductive construction to get a $L \otimes M$-frame for $\phi$. We will build a sequence $\left\langle\left\langle g_{i}, \beta_{i}, s\right\rangle \mid i \in \omega\right\rangle$ of frames which will be stationary for $i \geq a d p^{\square}(\phi)$. The construction of the models shall satisfy the following conditions, which we spell out for $i=2 k$; for odd indices the conditions are dual.

$[\mathbf{a}]_{2 k} g_{2 k}, \beta_{2 k}, s=\phi_{2 k}$

$[\mathbf{b}]_{2 k} \operatorname{dom}\left(\beta_{2 k}\right)=\operatorname{var}\left(\mathbb{S}_{2 k}^{\square}\right)$

$[\mathbf{c}]_{2 k}\left\langle g_{2 k}, \triangleleft_{2 k}\right\rangle=\left\langle g_{2 k-2}, \triangleleft_{2 k-2}\right\rangle \oplus h$ for some $h$, and $\triangleleft_{2 k}=\triangleleft_{2 k-1}$

$[\mathbf{d}]_{2 k} \quad g_{2 k}=L$

$[\mathbf{e}]_{2 k}$ For $x \in g_{2 k-1}$ :

(1) $\beta_{2 k}(p, x) \quad=\beta_{2 k-1}(p, x), \quad p \in \operatorname{var}(\phi)$

(2) $\beta_{2 k}(q \square, x) \leq \beta_{2 k-1}(\mathbf{\square}, x), \quad q \square \in \operatorname{var}\left(\mathbb{S}_{2 k}\right)$

(3) $\beta_{2 k-1}\left(q_{\square \psi}, x\right) \leq \beta_{2 k}\left(\square \psi^{\square}, x\right), \quad q_{\square \psi} \in \operatorname{var}\left(\mathbb{S}_{2 k-1}^{\square}\right)$

$[\mathbf{f}]_{2 k} X^{2 k}(x):=X_{\mathbf{a}_{2 k}, \mathbb{S}_{2 k}}^{\beta}(x)$ is consistent and sf-founded for $x \in g_{2 k}-g_{2 k-1}$

We begin the construction as follows. Since $\phi$ is $L \otimes M$-consistent, so is $\square^{\left(d g^{\square}(\phi)\right)} \Sigma_{\square}(\phi)^{\square} \wedge$ $\phi^{\square}$ because $\Sigma_{\square}(\phi)$ is a theorem of $L \otimes M$. A fortiori, $\square^{\left(d g^{\square}(\phi)\right)} \Sigma_{\square}(\phi)^{\square} \wedge \phi^{\square}$ is $L$-consistent and has a model 


$$
\left\langle g_{0}, \triangleleft_{0}\right\rangle, \gamma_{0}, s \models \square^{\left(d g^{\square}(\phi)\right)} \Sigma_{\square}(\phi)^{\square} ; \phi^{\square}
$$

with $\operatorname{dom}\left(\gamma_{0}\right)=\operatorname{var}\left(\mathbb{S}_{0}^{\square}\right)$. We may assume that $g_{0}=\operatorname{Tr}^{\square}\left(s, g_{0}\right)$. Now put $\beta_{0}\left(q_{\psi}, x\right)=*$ if $d g^{\square}(\psi)+\operatorname{dist}^{\square}(s, x)>d g^{\square}(\phi)$ and $\beta_{0}\left(q_{\psi}, x\right)=\gamma_{0}\left(q_{\psi}, x\right)$ else. In addition, $\beta_{0}(p, x)=*$ if $\operatorname{dist}^{\square}(s, x)>d g^{\square}(\phi)$. Then, by the above remark $\phi^{\square}$ is defined at $s$ and since $\beta_{0} \leq \gamma_{0}$

$$
\left\langle g_{0}, \triangleleft_{0}\right\rangle, \beta_{0}, s \models \phi^{\square}=\phi_{0}
$$

Therefore, $[\mathrm{a}]_{0}$ and $[\mathrm{d}]_{0}$ hold. For $[\mathrm{f}]_{0}$ note that $X^{0}(x) \subseteq X^{\gamma_{0}}(x)$; and the latter is consistent. And $X^{0}(x)$ is sf-founded since $\mathbb{S}_{0}$ is sf-founded and thus it is enough to see that $(\ddagger)$ if $\chi \in X^{0}(x), \tau \in s f(\chi)$ then also $\beta_{0}(\tau, x) \neq *$. This is, however, immediate; for $d g^{\square}(\chi)+\operatorname{dist}^{\square}(s, x) \leq d g^{\square}(\phi)$ implies $d g^{\square}(\tau)+\operatorname{dist}^{\square}(s, x) \leq d g^{\square}(\phi)$.

The inductive step is done only for the case $i=2 k>0$. For odd $i$ the construction is dual. Assume $[\mathrm{a}]_{2 k^{-}}[\mathrm{f}]_{2 k}$. For every point $t \in g_{2 k}-g_{2 k-1}$ we build a model

$$
\left\langle h_{t}, \boldsymbol{\triangleleft}\right\rangle, \gamma_{t}, t=\mathbf{\square}^{\left(d g \boldsymbol{\bullet}\left(\chi^{2 k}(t)\right)\right.} \Sigma_{\mathbf{\square}}\left(\chi^{2 k}(t)\right) ; \chi^{2 k}(t) \boldsymbol{\square}
$$

with $\chi^{2 k}(t):=\chi_{\mathbf{\square}^{\boldsymbol{q}_{2 k}}, \mathbb{S}_{2 k}}(t)$. This is possible since all the characteristic formulae are $L \otimes M$ consistent and so their $\mathbf{\square}$-ersatz is $M$-consistent. We assume that $h_{t} \cap h_{t^{\prime}}=\emptyset$ for $t \neq t^{\prime}$

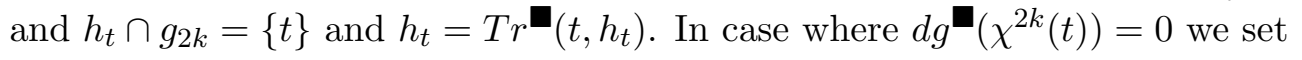

$$
\begin{aligned}
h_{t} & =\{t\} & & \\
\boldsymbol{\triangleleft}_{t} & =\{\langle t, t\rangle\} & & \text { if } \bullet \models M \\
& =\emptyset & & \text { else }
\end{aligned}
$$

Clearly then $\beta_{2 k}(q, t)=\gamma_{t}(q, t)$ for $q \in \operatorname{var}\left(\mathbb{S}_{2 k}\right)$. We put $\beta_{t}(q, x)=*$ for $q \notin \operatorname{var}\left(X^{2 k}(t)\right)$ and $\beta_{t}\left(q_{\square \psi}, x\right)=*$ if $d g \mathbf{\square}_{(\square \psi)+\operatorname{dist}(t, x)>d g}\left(\chi^{2 k}(t)\right)$; finally, $\beta_{t}(p, x)=*$ if dist $(t, x)>$ $d g\left(\chi^{2 k}(t)\right)$. But in all other cases $\beta_{t}(q, x)=\gamma_{t}(q, x)$. Clearly, $\beta_{t} \leq \gamma_{t}$. Now observe that $\operatorname{var}\left(\mathbb{S}_{2 k}\right)=\operatorname{var}\left(\mathbb{S}_{2 k+1}^{\mathbb{W}}\right)$ and therefore $\operatorname{var}\left(\chi^{2 k}(t) \subseteq \operatorname{var}\left(\mathbb{S}_{2 k+1}\right)\right.$. We can conclude that (1) $\chi^{2 k}(t)$ is defined at $t$ in $\left\langle h_{t}, \beta_{t}\right\rangle$ and therefore $\left\langle h_{t}, \boldsymbol{\triangleleft}_{t}\right\rangle, \beta_{t}, t=\chi^{2 k}(t)$ and that $(2)$ $X^{\beta_{t}}(x)$ is consistent and sf-founded (using $\left.(\ddagger)\right)$. Now let

$$
\begin{aligned}
& g_{2 k+1}=g_{2 k} \cup \bigcup\left\langle h_{t} \mid t \in g_{2 k}-g_{2 k-1}\right\rangle \\
& \triangleleft_{2 k+1}=\triangleleft_{2 k} \\
& \triangleleft_{2 k+1}=\triangleleft_{2 k} \cup \bigcup\left\langle\triangleleft_{t} \mid t \in g_{2 k}-g_{2 k-1}\right\rangle
\end{aligned}
$$

Define $\beta_{2 k+1}$ by $\beta_{2 k+1}(q, x):=\beta_{t}(q, x)$ for $x \in h_{t}$ and $\beta_{2 k+1}(q, x):=\beta_{2 k-1}(q, x)$ for $x \in g_{2 k-1}, q \in \operatorname{var}\left(\mathbb{S}_{2 k+1}\right)$; in all other cases $\beta_{2 k+1}(q, x)=*$. By construction, $[\mathrm{b}]_{2 k+1}$ 
holds. [c] $]_{2 k+1}$ holds by $\left\langle g_{2 k+1}, \boldsymbol{\iota}_{2 k+1}\right\rangle=\left\langle g_{2 k-1}, \boldsymbol{\iota}_{2 k-1}\right\rangle \oplus \bigoplus\left\langle h_{t} \mid t \in g_{2 k}-g_{2 k-1}\right\rangle$ and $\triangleleft_{2 k+1}=\triangleleft_{2 k} . \quad[\mathrm{d}]_{2 k+1}$ is immediate from $[\mathrm{c}]_{2 k+1},[\mathrm{~d}]_{2 k-1}$ and $h_{t}=M$. Now we show $[\mathrm{e}]_{2 k+1}$. Ad (1). Let $x \in g_{2 k-1}$. Then by $[\mathrm{e}]_{2 k} \beta_{2 k}(p, x)=\beta_{2 k-1}(p, x)=\beta_{2 k+1}(p, x)$. But if $x \in g_{2 k}-g_{2 k-1}$ then

$$
\begin{array}{ll} 
& \beta_{2 k+1}(p, x)=1 \\
\Leftrightarrow & \beta_{x}(p, x)=1 \\
\Leftrightarrow^{\dagger} & p \in X^{2 k}(x) \\
\Leftrightarrow & \beta_{2 k}(p, x)=1
\end{array}
$$

where $\Leftrightarrow^{\dagger}$ is true since $X^{2 k}(x)$ is sf-founded and $\operatorname{dom}\left(\beta_{x}\right)=\operatorname{var}\left(X^{2 k}(x)\right)$. Similarly, $\beta_{2 k+1}(p, x)=0 \Leftrightarrow \beta_{2 k}(p, x)=0$ is shown. Ad (2). If $x \in g_{2 k-1}$ we have $\beta_{2 k+1}\left(q_{\square \psi}, x\right)=$ $\beta_{2 k-1}\left(q_{\square \psi}, x\right) \leq \beta_{2 k}\left(\square \psi^{\square}, x\right)$ by $[\mathrm{e}]_{2 k}$. But if $x \in g_{2 k}-g_{2 k-1}$ then

$$
\begin{aligned}
& \beta_{2 k+1}\left(q_{\square \psi}, x\right)=1 \\
\Leftrightarrow & \beta_{x}\left(q_{\square \psi}, x\right)=1 \\
\Leftrightarrow & \square \psi \in X^{2 k}(x) \\
\Leftrightarrow & \beta_{2 k}\left(\square \psi^{\square}, x\right)=1
\end{aligned}
$$

and the argument continues as in (1). Ad (3). If $x \in g_{2 k-1}$ the claim follows by [e] $]_{2 k}$. If $\beta_{2 k}(q, x)=*$ then there is nothing to show. However, if $\beta_{2 k}\left(q \mathbf{q}_{\psi}, x\right) \neq *$ then $\boldsymbol{\square} \psi \in X^{2 k}(x)$ or $\neg \boldsymbol{\nabla} \psi \in X^{2 k}(x)$ and thus

$$
\begin{array}{ll} 
& \beta_{2 k+1}(\mathbf{\square}, x)=1 \\
\Leftrightarrow & \beta_{x}(\mathbf{\square}, x)=1 \\
\Leftrightarrow & \mathbf{\square} \psi X^{2 k}(x) \\
\Leftrightarrow & \beta_{2 k}(q \square, x)=1
\end{array}
$$

$[\mathrm{f}]_{2 k+1}$ holds because of $[\mathrm{c}]_{2 k+1}$ and by the definition of $\beta_{2 k+1}$ and finally because of (2) of $[\mathrm{e}]_{2 k+1}$. [a $]_{2 k+1}$ follows directly from $[\mathrm{e}]_{2 k+1}(1)$ and (3).

If $n=a d p^{\square}(\phi)$ we have $g_{n+1}=g_{n}$ and $d g \mathbf{\square}^{(}\left(\chi^{n}(t)\right)=d g^{\square}\left(\chi^{n}(t)\right)=0$ for all $t$ since $\mathbb{S}_{n}=\operatorname{var}(\phi)$ and therefore $\operatorname{dom}\left(\beta_{n}\right)=\operatorname{var}(\phi)$ by $[\mathrm{b}]_{n}$. By construction of the $h_{t}$, the $h_{t}$ are based on a single point and thus $g_{n+1}$ does not contain more points than $g_{n}$. Moreover, by $[\mathrm{d}]_{n},[\mathrm{~d}]_{n+1}, g_{n+1} \models L \otimes M$ and by $[a]_{n+1}, g_{n+1}, \beta_{n+1}, s \models \phi_{n+1}=\phi$. Take any valuation $\gamma \geq \beta_{n+1}$ which is standard for the $p$-variables. Then $g_{n+1}, \gamma, s=\phi$. $\dashv$

A few remarks on the completeness proof. First, Fine and Schurz [91] use a proof which is based on the same intuition. It is perhaps worthwile reading the explanations of the method in this paper. The fact that we use surrogate variables $q_{\psi}$ rather than the formulas they stand in for seems to complicate matters for the completenss proof; however, it will pay off when we prove results on interpolation and Halldén-completeness. Second, 
although we use the word 'construction' in the proof, the method for obtaining such a model is not constructive even when both $L$ and $M$ admit an effective construction of models (say, via tableaus). For the proof methods relies essentially on the consistency formulas which themselves can be constructed only when both $L$ and $M$ are decidable. We will return to this problem shortly.

Theorem 10 Suppose that $\perp \notin L, M$. Then $L \otimes M$ has f.m.p. iff both $L$ and $M$ have f.m.p.

The proof of this theorem is exactly the same, except that each partial model can be based on a finite frame. Since the construction terminates after finitely many steps, the resulting model is finite. The proof of Theorem 7 has a noteworthy consequence.

Corollary 11 Suppose that $\perp \notin L, M$ and that both logics are complete. Then

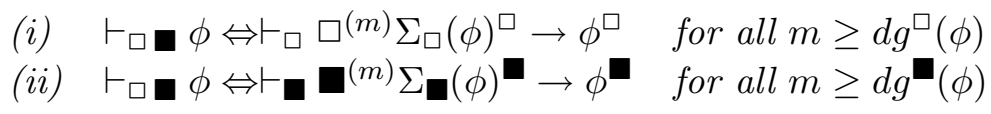

\section{Compactness is Invariant under Fusion}

A $\operatorname{logic} L$ is called compact if $\Phi \vdash \phi$ holds in $L$ iff for all $L$-frames $g$, valuations $\beta$ and points $x$

$$
g, \beta, x \models \Phi \Rightarrow g, \beta, x \models \phi
$$

Equivalently, $L$ is compact iff every consistent set has a model based on a frame for $L$. Compactness is therefore a much stronger property than completeness; every $\mathcal{D}$-persistent logic is compact.

Theorem 12 Let $\perp \notin L, M$. Then $L \otimes M$ is compact iff both $L$ and $M$ are compact.

Proof. Suppose $\Phi$ is an $L \otimes M$-consistent set of formulae. Use the same construction as in the proof of Theorem 7 with sets formulae and consistency sets rather than consistency formulae. The construction terminates after finitely many steps iff there is a bound for the alternation depth of the formulas in $\Phi$. If it terminates one can reason as before; however, if it does not then put $g=\bigcup\left\langle g_{i} \mid i \in \omega\right\rangle$. Then $g \models L$ and $g \models M$ by $[c]$ and $[d]$. For if $x \in g_{i}$ then $g, x=L \Leftrightarrow g_{i+1}, x=L$ and $g, x \models M \Leftrightarrow g_{i+1}, x \models M$. Both is the case. Hence $g \models L \otimes M$. For the valuation observe that $\beta_{i+1}(p, x)=\beta_{i}(p, x)$ for $x \in g_{i}$. And so we put $\beta(p, x)=\beta_{i}(p, x)$. Take any standard valuation $\gamma \geq \beta$. Then $g, \gamma, s=\Phi$. For if $\phi \in \Phi$ then for some $n, \phi_{n}=\phi$. Then $g_{n}, \beta, s=\phi$ and therefore $g, \beta, s \models \phi$ from which $g, \gamma, s=\phi . \dashv$ 
In presence of compactness it is actually possible to give a proof ot the theorem not using partial models. At each step we just require a model for $X^{2 k+1}(t)^{\square} ; \square^{(\omega)} \Sigma_{\square}\left(X^{2 k+1}(t)\right)$ and $X^{2 k}(t) ; \square^{(\omega)} \Sigma_{\square}\left(X^{2 k}(t)\right)$ respectively, where $\square^{(\omega)} \Phi$ denotes the set $\left\{\square^{k} \phi \mid k \in \omega, \phi \in \Phi\right\}$. The model $\left\langle h_{t}, \beta_{t}, t\right\rangle$ can be assumed to be generated by $t$ and therefore $X^{\beta_{t}}(x)$ is $L \otimes M$ consistent for all $x \in h_{t}$.

Now define a new consequence relation $\Vdash$; $\Phi \Vdash \phi$ holds iff $\phi$ can be derived from $\Phi$ and the axioms of the logic using Modus Ponens and Necessitation. If $L$ is a mono-modal logic then $\Phi \Vdash \phi$ iff $\square^{(\omega)} \Phi \vdash \phi$. $L$ is called $\Vdash$-complete if $\psi \Vdash \phi$ holds iff for all $L$-frames $g$ and valuations $\beta$

$$
g, \beta \models \psi \Rightarrow g, \beta \models \phi .
$$

If we say that $L$ is weakly compact if $\square^{(\omega)} \phi$ is consistent iff it has a model based on a $L$ frame then it is easily proved that $L$ is $\Vdash$-complete iff $\square^{(\omega)} \psi ; \phi$ has a Kripke-model (in the usual sense) exactly if it is consistent. Then $\Vdash$-completeness implies weak compactness. Finally, $L$ is $\Vdash$-compact if $\square^{(\omega)} \Phi ; \phi$ is consistent iff it has a model based on a $L$-frame. The following implications hold

$\begin{array}{ccc}\text { compact } & \Rightarrow & \text { complete } \\ \Downarrow & & \Uparrow \\ \Vdash \text {-compact } & \Rightarrow & \Vdash \text {-complete }\end{array}$

Theorem 13 Suppose $\perp \notin L, M$. Then $L \otimes M$ is $\Vdash$-complete ( $\Vdash$-compact, weakly compact) iff both $L$ and $M$ are $\Vdash$-complete $(\Vdash$-compact, weakly compact). $\dashv$

In Fine [74] a logic is called weakly compact if every consistent set based on finitely many variables has a Kripke-model on a frame for this logic. For this notion of weak compactness our method fails to yield a transfer theorem. Indeed, a counterexample can be contructed as follows. Take a mono-modal logic $L$ which is weakly compact but not compact. We show that $L \otimes \mathbf{K}$ is not weakly compact. For there exists a set $X$ which is $L$-consistent but lacks a Kripke-model. Then $X$ is based on infinitely many variables, namely $\operatorname{var}(X)=\left\{p_{i} \mid i \in \omega\right\}$; now let $\widetilde{X}$ result from $X$ by replacing the variable $p_{i}$ by the formula $\boldsymbol{\square}^{i} p$ for each $i \in \omega$. Then $\operatorname{var}(\widetilde{X})=\{p\}$ and so $\widetilde{X}$ is based on finitely many variables. Clearly, $\widetilde{X}$ is consistent; but if $\widetilde{X}$ has a model based on a Kripke-frame then this allows a direct construction of a Kripke-model for $X$. Thus $L \otimes \mathbf{K}$ is indeed not weakly compact. Everything hinges therefore on the existence of a weakly compact logic which is not compact. G.3 is such a logic. G.3 is weakly compact since it is of finite width, but is not compact by a result of Fine [85]. 


\section{Decidability}

Recall that a logic $L$ is decidable iff both $L$ and its complement $\mathcal{L}-L$ are recursively enumerable iff there is an effective algorithm deciding whether or not $\phi \in L$ for given $\phi$.

Theorem 14 Suppose that $\perp \notin L, M$ and that both logics are complete. Then $L \otimes M$ is decidable if both $L$ and $M$ are decidable.

Proof. By induction on $n:=a d p(\phi)$. If $n=0, \phi$ is boolean and since $\perp \notin L \otimes M$ $\vdash_{\square} \phi$ iff $\phi$ is a boolean tautology. Since the propositional calculus is decidable, this case is settled. Now suppose that for all $\psi$ with $a d p(\psi)<n$ we have shown the decidability of $\vdash_{\square} \boldsymbol{\square}$. We know by Corollary 11 that for $m \geq d g^{\square}(\phi), d g \mathbf{\square}_{(\phi)}$

$$
\begin{aligned}
& \vdash_{\square} \phi \Leftrightarrow \vdash_{\square} \square^{(m)} \Sigma_{\square}(\phi)^{\square} \rightarrow \phi^{\square} \\
& \vdash_{\square} \phi \Leftrightarrow \vdash^{(m)} \Sigma_{\square}(\phi)^{\square} \rightarrow \phi
\end{aligned}
$$

Therefore we can decide $\vdash_{\square} \phi$ on the condition that either $\Sigma_{\square}(\phi)$ or $\Sigma_{\boldsymbol{\square}}(\phi)$ can be constructed. But now either $\operatorname{adp}\left(\Sigma_{\square}(\phi)\right)<n$ or $\operatorname{adp}\left(\Sigma_{\mathbf{\square}}(\phi)\right)<n$. This is seen as follows. Suppose that $a d p^{\square}(\phi) \leq a d p \boldsymbol{\square}_{(\phi)}$. Then there is a maximal chain of nested alternating modalities starting with $\square$. Then any maximal chain of nested alternating modalities in $\Sigma_{\square}(\phi)$ starts with (!) and is a subchain of of such a chain in $\phi$. Consequently, $\operatorname{adp}\left(\Sigma_{\square}(\phi)\right)<a d p \square^{(\phi)}$ and with $\operatorname{adp} p^{\square}\left(\Sigma_{\square}(\phi)\right) \leq a d p^{\square}(\phi)$ the claim follows. Now let $\operatorname{adp}\left(\Sigma_{\square}(\phi)\right)<\operatorname{adp}(\phi)$ be the case. Then

$$
\Sigma_{\square}(\phi)=\bigvee\left\langle\psi_{c} \mid c \subseteq C, \nvdash_{\square} \sim \neg \psi_{c}\right\rangle
$$

Consequently, $\Sigma_{\square}(\phi)$ can be constructed if only $\vdash_{\square} \neg \psi_{c}$ is decidable for all $c$. But this is so because $\operatorname{adp}\left(\neg \psi_{c}\right)<n$. $\dashv$

Note that for $m>1 a d p^{\square}\left(\square^{(m)} \Sigma_{\square}(\phi)\right) \leq a d p^{\square}(\phi)$ but $a d p \mathbf{\square}_{\left(\square^{(m)} \Sigma_{\square}(\phi)\right) \leq a d p} \mathbf{\square}_{(\phi)+}$ 1. A case where the inequalities are sharp is given by $\phi=\mathbf{\square}_{p}$. But in all these cases $a d p^{\square}(\phi)>\operatorname{adp}(\phi)$ in which case we also have $a d p^{\square}\left(\boldsymbol{\square}^{(m)} \Sigma_{\mathbf{m}}(\phi)\right) \leq a d p^{\square}(\phi)$ and $\operatorname{adp}\left(\Sigma_{\mathbf{\square}}(\phi)\right)<\operatorname{adp}{ }^{\square}\left(\boldsymbol{\square}^{(m)} \Sigma_{\mathbf{\square}}(\phi)\right) \leq a d p^{\square}(\phi)$ and therefore $\operatorname{adp}\left(\boldsymbol{\square}^{(m)} \Sigma_{\mathbf{\square}}(\phi)\right) \leq \operatorname{adp}(\phi)$. This will be needed later.

Decidability does not imply completeness. A counterexample is given in Creswell [84]. On the other hand, finite model property does not imply decidability since there are uncountably many logics with f.m.p. So these properties are clearly not linked in a straightforward way. 


\section{Interpolation and Halldén-completeness}

In this section we will show that interpolation and Halldén-completenss are both preserved under fusion provided that the two logics are complete. Recall that a logic $L$ is said to have interpolation if whenever $\phi \rightarrow \psi \in L$ there is a formula $\chi$ such that $\operatorname{var}(\chi) \subseteq$ $\operatorname{var}(\phi) \cap \operatorname{var}(\psi)$ and $\phi \rightarrow \chi, \chi \rightarrow \psi \in L . \chi$ is called an interpolant for $\phi$ and $\psi$. L is called Halldén-complete if whenever $\phi \vee \psi \in L$ and $\operatorname{var}(\phi) \cap \operatorname{var}(\psi)=\emptyset$ then $\phi \in L$ or $\psi \in L$. Equivalently, $L$ is Halldén-complete iff for $\phi$ and $\psi$ based on disjoint sets of variables $\phi \wedge \psi$ is $L$-consistent iff both $\phi$ and $\psi$ are $L$-consistent. Halldén-completeness is closely connected with the notion of relevance. According to a widely accepted definition, a logic is relevant if whenever $\Phi \vdash_{L} \phi$ and $\phi$ is not an $L$-theorem, then $\operatorname{var}(\Phi) \cap \operatorname{var}(\phi) \neq \emptyset$. A logic which is Halldén-complete is a logic which is as relevant as possible while still being classical. For $L$ is Halldén-complete iff $\Phi \vdash_{L} \phi$ for a nontheorem $\phi$ implies either that $\Phi$ is inconsistent or that $\operatorname{var}(\Phi) \cap \operatorname{var}(\phi) \neq \emptyset$. So, $L$ is relevant with the exception of "ex falso quodlibet".

For mono-modal logics, interpolation does not imply Halldén-completeness. For if $\phi \rightarrow \psi \in L$ and $\operatorname{var}(\phi) \cap \operatorname{var}(\psi)=\emptyset$ then by interpolation there is a constant formula $\chi$ such that $\phi \rightarrow \chi, \chi \rightarrow \psi \in L$; we cannot, however, conclude $\neg \phi \in L$ or $\psi \in L$. This is only the case if $\chi$ is either $T$ or $\perp$. For a counterexample take $\square \perp \rightarrow \square \perp$ (see van Benthem and Humberstone [83]). In fact, if $L$ is Halldén-complete then either $L \supseteq K(\mathrm{x})$ or $L \supseteq \mathbf{K} / \mathbf{x}=\mathbf{K}(\diamond \top)$. And under the same conditions interpolation implies Halldéncompleteness. Thus, while $\mathbf{K}$ and $\mathbf{K} \mathbf{4}$ have interpolation, they are not Halldén-complete. Also, van Benthem and Humberstone [83] show that $\mathbf{S 4 . 3}$ is Halldén-complete; but it lacks interpolation as is shown by L. L. Maximova (see Rautenberg [83]).

Classical (propositional) logic has interpolation and is therefore also Halldén-complete. Rautenberg [83] proves that if a logic allows tableaus of a certain type then this logic has interpolation. These results can be boosted up to multi-modal logics. For if $L$ and $M$ are two logics which admit such tableaus, then the rules of $L \otimes M$ are just the rules for $L$ and $M$ together. Obviously, in this case interpolation for $L \otimes M$ is proved and the resulting tableau has the additional virtue to allow a direct computation of the interpolant. In the general case considered here, such a direct method is not available. However, if both $L$ and $M$ are decidable and each not only has interpolation but also allows an effective construction of an interpolant then $L \otimes M$ has all these properties as well since we will give a construction of the interpolant, which is effective under these circumstances.

The proof in both cases consists in a close analysis of the consistency formulae $\Sigma_{\square}(\phi \vee \psi)$ and $\Sigma_{\square}(\phi \rightarrow \psi)$. Since both are identical, it suffices to concentrate on the latter. We can write $\Sigma_{\square}(\phi)=\bigvee\left\langle\widetilde{\phi}_{c} \mid c \in C\right\rangle$ and $\Sigma_{\square}(\psi)=\bigvee\left\langle\widetilde{\psi}_{d} \mid d \in D\right\rangle$. Then obviously $\Sigma_{\square}(\phi \rightarrow \psi)$ is (up to boolean equivalence) a suitable disjunction of $\widetilde{\phi}_{c} \wedge \widetilde{\psi}_{d}$; namely, this disjunction is taken over the set $E$ of all pairs $\langle c, d\rangle$ such that $\widetilde{\phi}_{c} \wedge \widetilde{\psi}_{d}$ is consistent. Equivalently, we can write

$$
\Sigma_{\square}(\phi \rightarrow \psi)=\Sigma_{\square}(\phi) \wedge \Sigma_{\square}(\psi) \wedge \bigwedge\left\langle\widetilde{\phi}_{c} \rightarrow \neg \widetilde{\psi}_{d} \mid\langle c, d\rangle \notin E\right\rangle
$$


We abbreviate the third conjunct by $\nabla(\phi ; \psi)$ (or, to be more precise we would again have to write $\left.\nabla_{\square}(\phi ; \psi)\right)$. Obviously, $\nabla(\phi ; \psi)$ serves to cut out the unwanted disjuncts. In some sense $\nabla(\phi ; \phi)$ measures the extent to which $\phi$ and $\psi$ are interdependent. So if $\nabla(\phi ; \psi)=\top$ both are independent. It is vital to observe that all reformulations are classical equivalences.

Theorem 15 Suppose that $\perp \notin L, M$ and that both logics are complete. Then $L \otimes M$ is Halldén-complete iff both $L$ and $M$ are.

Proof. $(\Rightarrow)$ Suppose $\phi \vee \psi \in L$ and $\operatorname{var}(\phi) \cap \operatorname{var}(\psi)=\emptyset$. Then $\phi \vee \psi \in L \otimes M$ and so either $\phi \in L \otimes M$ or $\psi \in L \otimes M$ and thus either $\phi \in L$ or $\psi \in L$, since $L \otimes M$ is a conservative extension of $L$.

$(\Leftarrow)$ By induction on $n=a d p(\phi \vee \psi)$. For $n=0$ this follows from classical logic. Now assume that $n>0$ and that the theorem is proved for all formulae of alternation depth $<n$. Take $\phi \vee \psi$ such that $\operatorname{var}(\phi) \cap \operatorname{var}(\psi)=\emptyset$ and $a d p(\phi \vee \psi)=n$. Assume $a d p^{\square}\left(\Sigma_{\square}(\phi \vee \psi)\right)<$ $a d p^{\square}(\phi \vee \psi)$. Then by Corollary 11, $\vdash_{\square} \square^{(m)} \Sigma_{\square}(\phi \vee \psi)^{\square} \rightarrow . \phi^{\square} \vee \psi^{\square}$ for large $m$, by which

$$
\vdash_{\square} \square^{(m)} \Sigma_{\square}(\phi)^{\square} \wedge \square^{(m)} \Sigma_{\square}(\psi)^{\square} \wedge \square^{(m)} \nabla(\phi ; \psi)^{\square} \rightarrow . \phi^{\square} \vee \psi^{\square}
$$

The crucial fact now is that $\nabla(\phi ; \psi)=\top$. For if $\widetilde{\phi}_{c}$ and $\widetilde{\psi}_{d}$ are both $L \otimes M$-consistent, then since $\operatorname{var}\left(\widetilde{\phi}_{c}\right) \cap \operatorname{var}\left(\widetilde{\psi}_{d}\right) \subseteq \operatorname{var}(\phi) \cap \operatorname{var}(\psi)=\emptyset$ and $\operatorname{adp}\left(\widetilde{\phi}_{c}\right), a d p\left(\widetilde{\psi}_{d}\right)<n, \widetilde{\phi}_{c} \wedge \widetilde{\psi}_{d}$ is $L \otimes M$-consistent by induction hypothesis. Thus $\nabla(\phi ; \psi)$ is an empty conjunction. Consequently, we can rewrite the above to

$$
\begin{aligned}
& \vdash_{\square} \square^{(m)} \Sigma_{\square}(\phi)^{\square} \wedge \square^{(m)} \Sigma_{\square}(\psi)^{\square} \rightarrow . \phi^{\square} \vee \psi^{\square} \\
& \vdash_{\square} \square^{(m)} \Sigma_{\square}(\phi)^{\square} \rightarrow \phi^{\square} . \vee . \square^{(m)} \Sigma_{\square}(\psi)^{\square} \rightarrow \psi^{\square}
\end{aligned}
$$

Now since $L$ is Halldén-complete, we have $\square^{(m)} \Sigma_{\square}(\phi)^{\square} \rightarrow \phi^{\square} \in L$ or $\square^{(m)} \Sigma_{\square}(\psi)^{\square} \rightarrow \psi^{\square} \in$ $L$ from which by Corollary $11 \phi \in L \otimes M$ or $\psi \in L \otimes M$. $\dashv$

Theorem 16 Suppose that $\perp \notin L, M$ and that both logics are complete. Then $L \otimes M$ has interpolation iff both $L$ and $M$ have interpolation. Moreover, if $\phi \rightarrow \psi \in L \otimes M$ then an interpolant $\chi$ can be found such that $a d p^{\square}(\chi) \leq \min \left\{a d p^{\square}(\phi), a d p^{\square}(\psi)\right\}$ and $a d p \cdot(\chi) \leq \min \{a d p \cdot(\phi), a d p \cdot(\psi)\}$.

Proof. $(\Rightarrow)$ Let $\phi \rightarrow \psi \in L$. Then by hypothesis there is a $\chi$ such that $\phi \rightarrow \chi, \chi \rightarrow \psi \in$ $L \otimes M$ based on the common variables of $\phi$ and $\psi$. Now, by Makinson's Theorem, either $M(p \leftrightarrow \square p)$ or $M(\mathbb{\square} p)$ is consistent. Let the former be the case. Then let $\chi^{\circ}$ result from $\chi$ by successively replacing a subformula $\psi$ by $\psi$. Then $\chi^{\circ} \in \mathcal{L}_{\square}$ and $\chi \leftrightarrow \chi^{\circ} \in M(p \leftrightarrow \mathbf{\square})$. Hence, as $\phi \rightarrow \chi \in L \otimes M$, then also $\phi \rightarrow \chi^{\circ} \in L \otimes M(p \leftrightarrow \mathbf{\square} p)$. But $L \otimes M(p \leftrightarrow \boldsymbol{\square} p)$ is a conservative extension of $L$ and therefore $\phi \rightarrow \chi^{\circ} \in L$. In the case where $M(\mathbb{\square} p)$ is consistent, define $\chi^{\circ}$ to be the result of replacing subformulas of type $\psi$ by $\top$. Then use the same argument as before. 
$(\Leftarrow)$ By induction on $n=a d p(\phi \rightarrow \psi)$. The case $n=0$ is covered by classical logic. Now suppose that $n>0$ and that the theorem has been proved for all formulae of alternation depth $<n$. Let $\phi \rightarrow \psi \in L \otimes M$. We may assume that $a d p^{\square}(\phi \rightarrow \psi) \leq \operatorname{adp}(\phi \rightarrow \psi)$ and thus $\operatorname{adp}\left(\Sigma_{\square}(\phi \rightarrow \psi)\right)<\operatorname{adp}(\phi \rightarrow \psi)$ (see the calculations following Theorem 14). Then $\operatorname{adp}\left(\Sigma_{\square}(\phi \rightarrow \psi)\right)<a d p(\phi \rightarrow \psi)$. By Corollary 11, for sufficiently large $m$,

$$
\begin{array}{ll} 
& \vdash_{\square} \square^{(m)} \Sigma_{\square}(\phi \rightarrow \psi) \rightarrow . \phi^{\square} \rightarrow \psi^{\square} \\
(\dagger) \quad \vdash_{\square} \square^{(m)} \Sigma_{\square}(\phi)^{\square} \wedge \phi^{\square} \wedge \square^{(m)} \nabla(\phi ; \psi)^{\square} \rightarrow . \square^{(m)} \Sigma_{\square}(\psi)^{\square} \rightarrow \psi^{\square}
\end{array}
$$

Let $\widetilde{\phi}_{c} \rightarrow \neg \widetilde{\psi}_{d}$ be a conjunct of $\nabla(\phi ; \psi)$. By induction hypothesis and the fact that

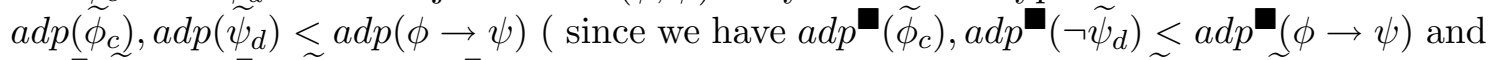
$\left.a d p^{\square}\left(\widetilde{\phi}_{c}\right), a d p^{\square}\left(\neg \widetilde{\psi}_{d}\right) \leq a d p^{\square}(\phi \rightarrow \psi)\right)$ there is an interpolant $Q_{c, d}$ for $\widetilde{\phi}_{c}$ and $\widetilde{\psi}_{d}$. Note that $\operatorname{var}\left(Q_{c, d}\right)=\operatorname{var}^{p}\left(Q_{c, d}\right) \subseteq \operatorname{var}(\phi) \cap \operatorname{var}(\psi)$ and that $a d p^{\square}\left(Q_{c, d}\right) \leq \min \left\{a d p^{\square}\left(\widetilde{\phi}_{c}\right), a d p^{\square}\left(\widetilde{\psi}_{d}\right)\right\}$ $\leq \min \left\{a d p^{\square}(\phi), a d p^{\square}(\psi)\right\}$ and likewise for $\boldsymbol{\square}$. Again by Corollary 11 we get

$$
\vdash_{\square} \square^{(m)} \Sigma_{\square}\left(\widetilde{\phi}_{c} \rightarrow Q_{c, d}\right)^{\square} \wedge \square^{(m)} \Sigma_{\square}\left(Q_{c, d} \rightarrow \neg \widetilde{\psi}_{d}\right)^{\square} . \rightarrow .\left(\widetilde{\phi}_{c} \rightarrow Q_{c, d}\right)^{\square} \wedge\left(Q_{c, d} \rightarrow \neg \widetilde{\phi}_{d}\right)^{\square}
$$

and therefore with $F=C \times D-E$ (recall the definition of $\nabla$ )

$$
\begin{gathered}
\bigwedge_{F} \square^{(m)} \Sigma_{\square}\left(\widetilde{\phi}_{c} \rightarrow Q_{c, d}\right)^{\square} \wedge \bigwedge_{F} \square^{(m)} \Sigma_{\square}\left(Q_{c, d} \rightarrow \neg \widetilde{\psi}_{d}\right)^{\square} \\
\quad \vdash_{\square} \bigwedge_{F}\left(\widetilde{\phi}_{c} \rightarrow Q_{c, d}\right)^{\square} \wedge \bigwedge_{F}\left(Q_{c, d} \rightarrow \neg \widetilde{\psi}_{d}\right)^{\square} \\
\quad \vdash_{\square} \nabla(\phi ; \psi)^{\square}
\end{gathered}
$$

Thus $(\dagger)$ can be rewritten modulo boolean equivalence to

$$
\begin{aligned}
& \square^{(m)} \Sigma_{\square}(\phi)^{\square} \wedge \phi^{\square} \wedge \bigwedge_{F} \square^{(m)} \Sigma_{\square}\left(\widetilde{\phi}_{c} \rightarrow Q_{c, d}\right)^{\square} \\
& \quad \vdash_{\square} \bigwedge_{F} \square^{(m)} \Sigma_{\square}\left(Q_{c, d} \rightarrow \neg \widetilde{\psi}_{d}\right)^{\square} \wedge . \square^{(m)} \Sigma_{\square}(\psi)^{\square} \rightarrow \psi^{\square}
\end{aligned}
$$

Abbreviate the formula to the left by $\eta_{\ell}$ and the one to the right by $\eta_{r}$. Then $a d p^{\square}\left(\eta_{\ell}^{\uparrow}\right)=$ $\max \left\{a d p^{\square}\left(\square^{(m)} \Sigma_{\square}(\phi), a d p^{\square}(\phi), a d p^{\square}\left(\bigwedge_{F} \square^{(m)} \Sigma_{\square}\left(\widetilde{\phi}_{c} \rightarrow Q_{c, d}\right)\right)\right\}=a d p^{\square}(\phi)\right.$ (since we have that $a d p^{\square}\left(\square^{(m)} \Sigma_{\square}(\phi)\right) \leq a d p^{\square}(\phi)$ by an earlier observation and $a d p^{\square}\left(\bigwedge_{F} \square^{(m)} \Sigma_{\square}\left(\widetilde{\phi}_{c} \rightarrow\right.\right.$ $\left.\left.\left.Q_{c, d}\right)\right) \leq a d p^{\square}\left(\square^{(m)} \Sigma_{\square}(\phi)\right)\right)$ and by a similar argument $a d p^{\square}\left(\eta_{r}^{\uparrow}\right)=\max \left\{a d p^{\square}\left(\square^{(m)} \Sigma_{\square}(\psi)\right)\right.$, $\left.a d p^{\square}(\psi), a d p^{\square}\left(\bigwedge_{F} \square^{(m)} \Sigma_{\square}\left(Q_{c, d} \rightarrow \neg \widetilde{\psi}_{d}\right)\right)\right\}=a d p^{\square}(\psi)$; and likewise for $a d p$. By assumption on $L$, there is an interpolant $\chi$ for $\eta_{\ell}$ and $\eta_{r}$. By definition, $\chi$ is based on the same surrogate variables as $\eta_{\ell}$ and $\eta_{r}$. Then for the total reconstruction $\chi^{\uparrow}$ of $\chi$ $a d p^{\square}\left(\chi^{\uparrow}\right) \leq \min \left\{a d p^{\square}\left(\eta_{\ell}^{\uparrow}\right), a d p^{\square}\left(\eta_{r}^{\uparrow}\right)\right\}=\min \left\{a d p^{\square}(\phi), a d p^{\square}(\psi)\right\}$ and likewise for $a d p$. It is easily verified that $\operatorname{var}^{p}\left(\chi^{\uparrow}\right) \subseteq \operatorname{var}^{p}(\phi) \cap \operatorname{var}^{p}(\psi)$. Moreover, from $\eta_{\ell}=\eta_{\ell}^{\uparrow \square} \vdash_{\square} \chi^{\uparrow \square}$ with Corollary 11 and the fact that the consistency formulae are $L \otimes M$-theorems we conclude that $\phi \vdash_{\square} \chi^{\uparrow}$ and likewise that $\chi^{\uparrow} \vdash_{\square} \boldsymbol{\square} \psi \dashv$

Theorem 16 implies an even stronger interpolation property for $L \otimes M$. Namely, if $\phi \rightarrow$ $\psi \in L \otimes M$ then an interpolant exists which is not only based on the common variables but also contains only the modalities which occur in both $\phi$ and $\psi$. 
Relatively little is known about the connection between completeness and interpolation and Halldén-completeness. These are probably independent properties. $\mathbf{S 4 . 3}$ has f.m.p. but lacks interpolation. On the other hand, if we define $\mathbf{K} \mathbf{4}_{\omega}$ to be the extension of $\mathbf{K} \mathbf{4}$ by all constant formulae which are theorems of $\mathbf{G}$ then it can be shown that $\mathbf{K} \mathbf{4}_{\omega}$ has interpolation (Rautenberg [83]) and $\mathbf{K} \mathbf{4}_{\omega}$ lacks f.m.p. (Kracht [91]).

\section{Outlook}

We should stress again that the results we have obtained so far generalize to logics with arbitrary many modal operators - even infinitely many. For persistence this is straightforward, but in the case of other proerties some care has to be exercised. For example with f.m.p., it is possible to redo the our proof using the same construction except that it now has to cycle between all of the modalities. If there only finitely many of them, this construction stays finite. If there are infinitely many, we build first a model based on only those modalities actually occurring in $\phi$ and then use a poly-modal analogue of Corollary 2 to obtain a model on the same set of worlds for the other modalities. Another possibility is to show that if $M$ and $N$ are arbitrary $m$ - $/ n$-modal logics then $M \otimes N$ has a property $P$ iff both $M$ and $N$ have this property. In fact, the second author has recently shown that all the theorems can be generalized in this way with the exception that it cannot be proved that interpolation of $M \otimes N$ implies interpolation for $M$ and $N$ although the converse still holds.

For logics which are not independently axiomatizable the situation is of course more complicated. We did not succeed in showing that for any mono-modal logic $L$ its minimal tense extension $L t=L \otimes \mathbf{K}(p \rightarrow \square \vee p, p \rightarrow \mathbf{\square} \diamond p)$ which is an extension of $L \otimes \mathbf{K}, \mathbf{K}$ the minimal logic, inherits the completeness properties of $L$ although this is a plausible guess. It does, however, inherit the persistence properties of $L$ since both $\mathbf{K}_{\square} \mathbf{\square}(p \rightarrow \square p)$ and $\mathbf{K}_{\square}(p \rightarrow \mathbf{\square} \diamond p)$ are $\mathcal{R}$-persistent and so also $\mathcal{D}$-persistent. On the positive side we have a result in Kracht [90] on the logic $\bigotimes_{i \in n} \mathbf{A l t}_{1} \otimes \mathbf{G r z}\left(\left\{\square_{n} p \rightarrow \square_{i} p \mid i \in n\right\}\right)$ with $\mathbf{A l t}_{1}=$ $\mathbf{K}(\diamond p \wedge \diamond q \rightarrow \diamond(p \wedge q))$ and $\mathbf{G r z}=\mathbf{K} \mathbf{4}(\square(\square(p \rightarrow \square p) \rightarrow p) \rightarrow p)$ which can be shown to have f.m.p. by showing that the addition of the axioms $\square_{n} p \rightarrow \square_{i} p$ preserves the finite model property of the base logic $\bigotimes_{i \in n} \mathbf{A l t}_{1} \otimes \mathbf{G r z}$.

Let us also add that using the techniques of Kracht [90] or Sambin and Vaccaro [89] the following generalization of Sahlqvist's theorem can be proved.

Theorem 17 Let $T$ be an n-modal formula which is equivalent to a conjunction of formulae of the form $\bar{P}\left(T_{1} \rightarrow T_{2}\right)$ where $\bar{P}$ is a prefix of modalities, $T_{2}$ is positive and $T_{1}$ is obtained from propositional variables and constants in such a way that no positive occurrence of a variable is in a subformula of the form $U_{1} \vee U_{2}$ or $\diamond_{i} U_{1}$ within the scope of some $\square_{j}$. Then $T$ is effectively equivalent to a first-order formula and $\mathbf{K}(T)$ is $\mathcal{D}$-persistent. 


\section{References}

[83] van Benthem, J. F. A. K., Humberstone, I. L.: Halldén-Completeness by Gluing Kripke Frames, NDJFL 24(1983), 426 - 430

[84] Cresswell, M.: An incomplete decidable logic, JSL 13(1984), 520 - 527

[74] Fine, K.: Logics containing K4, Part I, JSL 39(1974), 229 - 237

[85] Fine, K.: Logics containing K4, Part II, JSL 50(1985), 619 - 651

[91] Fine, K., Schurz, G.: Transfer theorems for stratified multimodal logics, to appear

[51] Jónsson, B., Tarski, A.: Boolean Algebras with Operators, Am. Journ. of Math. $73(1951), 891-939$

[89] Kracht, M.: On the Logic of Category Definitions, Computational Linguistics 15(1989), $111-113$

[90] Kracht, M.: Internal Definability and Completeness in Modal Logic, Doctoral Dissertation, Freie Universität Berlin, 1990

[91] Kracht, M.: Splittings and the Finite Model Property, to appear

[71] Makinson, D. C.: Some embedding theorems for modal logic, NDJFL 12(1971), 252 254

[83] Rautenbrg, W.: Modal tableau calculi and interpolation, JPL 12(1983), 403 - 423

[75] Sahlqvist, H.: First and second order semantics for modal logic, in: Kanger (ed.): Proceedings of the $3^{\text {rd }}$ Scandinavian Logic Symposium, North-Holland, Amsterdam, $1975,110-143$

[88] Sambin, G., Vaccaro, V.: Topology and duality in modal logic, Annals of Pure and Applied Logic 37(1988), 249 - 296

[89] Sambin, G., Vaccaro, V.: A topological proof of Sahlqvist's theorem, JSL 54(1989), $992-999$

[80] Thomason, S. K. Independent Propositional Modal Logics, Studia Logica 39(1980), $143-144$ 